Radiocarbon is available online to all subscribers (access is free!)

\title{
ingenta
}

\section{Benefits include:}

- Document-to-document linking via references for fast reliable access to the wider literature

- Fully searchable across full text, abstracts, text, titles, TOC and figures delivering comprehensive search results

- Links to and from major Abstract and Indexing resources to aid research

- Full-text searching across multiple journals for a wider view of the research that counts

- TOC alerting service keeping you up to date with the latest research

- Access full-text articles online before you receive the hard copy issue

\section{Set up Access now at:}

- www.ingentaselect.com/register.htm and follow the online instructions*

\section{Not a subscriber? Why not?}

Find out more by contacting

Mark McClure

Email: editor@radiocarbon.org

\section{Subscription Enquiries:}

support@ingenta.com

${ }^{*}$ Access is provided by Ingenta 


\section{ADVERTISE IN RADIOCARBON}

\section{REACH YOUR TARGET AUDIENCE EFFECTIVELY \& AFFORDABLY}

\begin{tabular}{lll}
\multicolumn{3}{l}{ Prices good through November 1,2005} \\
\hline \multicolumn{2}{l}{ Display Ads } \\
\hline Inside page & Inside back cover & Back cover \\
\$350/issue & $\$ 500 /$ issue & $\$ 600 /$ issue \\
$\$ 900 /$ volume & $\$ 1250 /$ volume & $\$ 1500 /$ volume \\
\hline 2Reserved through 2005. & \\
\hline & \\
\hline Color Ads and Folded Inserts & \\
\hline Color ad (inside page) & Folded inserts \\
\$1600/issue & $\$ 300 /$ issue \\
\$4500/volume & $\$ 700 /$ volume \\
\hline
\end{tabular}

aThe prices above are for prepared inserts and ads provided by the customer. Call for estimates on typesetting, design, duplicating and folding services.

Radiocarbon reaches libraries, labs, individuals, and institutions in North America, South America, Europe, the Middle East, Asia, Africa, and Australasia. We can help you reach your target market, wherever it is.

We publish three issues per volume year. Advertise in two consecutive issues and get the third at a significant discount (must reserve all three ads at once for discount to apply).

We accept full-page and multipage advertisements in black and white or full-color. You may send camera-ready copy, film negatives, or digital files. Or, for a small fee, we will create an ad from your supplied text and graphics.

For more information, please contact Managing Editor MarkMcClure at editor@radiocarbon.org. 


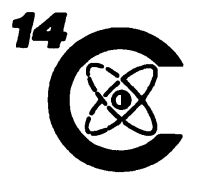

Radiocarbon

An International Journal of

Cosmogenic Isotope Research
Univ. of Arizona, Dept.of Geosciences 4717 E. Fort Lowell Rd, Rm. 104

Tucson, AZ 85712-1201 USA

Phone +1 520 881-0857

Fax: +1 520 881-0554

E-mail: orders@radiocarbon.org

http://www.radiocarbon.org

\section{PRICE LIST}

Proceedings of the 18th International Radiocarbon Conference

(Vol 46, Nrs 1 and 2, 2004) Save $\$ 10$

Proceedings of the 17th International Radiocarbon Conference $\quad 75.00$

(Vol 43, Nrs 2A, 2B and 3, 2001)

Proceedings of the 16th International Radiocarbon Conference $\quad 50.00$

(Vol 40, Nrs 1 and 2, 1998)

IntCal04 (2004 Calibration issue; Vol 46, Nr 3, 2004) 40.00

IntCal98 (1998 Calibration issue; Vol 40, $\mathrm{Nr} \mathrm{3,} \mathrm{1998)} 40.00$

$\begin{array}{lr}\text { Calibration } 1993 \text { (Vol 35, Nr 1, 1993) } & 40.00\end{array}$

Proceedings of the 15th International Radiocarbon Conference (Vol 37, $\mathrm{Nr} 2,1995) \quad 50.00$

Liquid Scintillation Spectrometry 1992 (ISBN 0-9638314-0-2; 1993) 20.00

Liquid Scintillation Spectrometry 1994 (ISBN 0-9638314-3-7; 1996) 10.00

Liquid Scintillation Spectrometry 2001 (ISBN 0-9638314-4-5; 2002) 60.00

Special offer-LSC 92, LSC 94, LSC 2001 package-save \$20.00

Tree Rings, Environment and Humanity (ISBN 0-9638314-2-9; 1996) 20.00

(Proceedings of the International Tree-Ring Conference, Tucson, Arizona, 1994)

Late Quaternary Chronology and Paleoclimates of the Eastern Mediterranean 40.00 (ISBN 0-9638314-1-0; 1994) Back in print!

SUBSCRIPTION RATES VOLUME 47, Nrs 1-3, 2005 (subscriptions include free online access)

$\begin{array}{ll}\text { Institution (US, shipping included) } & 200.00\end{array}$

$\begin{array}{lr}\text { Institution (Foreign, shipping included) } & 215.00\end{array}$

$\begin{array}{lr}\text { Individual (US, shipping included) } & 100.00\end{array}$

$\begin{array}{lr}\text { Individual (Foreign, shipping included) } & 115.00\end{array}$

$\begin{array}{lr}\text { Lifetime Subscription-Institutional } & 2500.00\end{array}$

$\begin{array}{lr}\text { Lifetime Subscription-Individual } & 1000.00\end{array}$

BACK ISSUES (except conference proceedings and special issues) $\quad$ Single issue 40.00

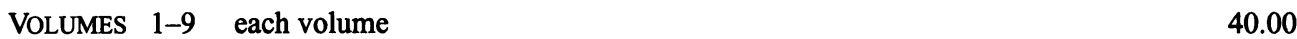

VOLUMES 10-21 each volume $\quad 65.00$

VOLUMES 22-45 each volume $\quad 100.00$

$\begin{array}{lr}\text { Radiocarbon Conference Proceedings } & 50.00\end{array}$

SPECIAL FULL-SET OFFER_Volumes 1-46 (1959-2004) 1300.00

Big savings! Includes bound copies of 35 out-of-print issues. Take $\$ 50.00$ off for each additional set.

\section{Postage ANd Handling ChaRT}

\begin{tabular}{l|c|r}
\hline & U.S. & \multicolumn{1}{|c}{ Foreign } \\
\hline Subscription & - & $\$ 15.00$ \\
\hline Single back issue & $\$ 4.00$ & $\$ 9.00$ \\
\hline Book or Proceedings & $\$ 5.00$ & $\$ 15.00$ \\
\hline Full set & $\$ 72.00$ & $\$ 400.00$ \\
\hline
\end{tabular}

Surface mail rates are listed here. Please contact us for airmail or express delivery rates.

Orders must be prepaid. We accept payments by Visa, MasterCard, or AMEX or by check or money order payable in US\$ to Radiocarbon. Bank funds transfers are also accepted. Please contact us for instructions.

*Postage will be added; see above chart. Subscription rates and book prices are subject to change. 


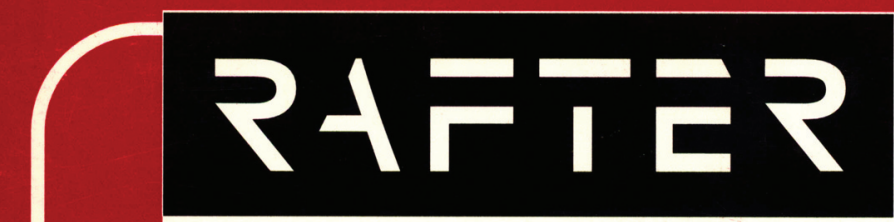

\section{RADIOCARBON LABORATORY}

\section{The test of time}

Accelerator Mass Spectrometry Radiocarbon Dating

Rafter provides:

$\downarrow$ Prompt reporting times

$\diamond$ never more than 8 weeks

4 Competitive prices

$\diamond N Z \$ 820$ per sample

$\downarrow$ Stable isotope corrections

$\diamond \delta^{13} \mathrm{C}$ measurement at no extra charge

$\downarrow$ Additional services

$\diamond 3$ week express turnarounds

$\diamond$ enhanced precision measurements

$\diamond$ radiometric ${ }^{14} \mathrm{C}$ dating for large samples

$\diamond$ bone $C: N$ analysis:- $\% C, \% N, \delta^{13} C, \delta^{15} N$

$\diamond$ direct dating of pollen

$\diamond X$-ray diffraction for shells

$\diamond$ calibration assistance

$\diamond$ consultancy services
At the Rafter Radiocarbon Laboratory we have been successfully meeting the test of time for more than 50 years.

The Rafter Radiocarbon Laboratory has an international reputation for accurately dating a wide range of organic materials, sediments, textiles, bone, ivory, paper, wood, parchment, charcoal, shell, foraminifera and peat.

At Rafter we understand what our clients expect: accurate dating, at competitive rates and superior turn around times and service.

\section{www.RafterRadiocarbon.co.nz}

\section{or contact us at:}

Rafter Radiocarbon Laboratory Institute of Geological \& Nuclear Sciences 30 Gracefield Road, Lower Hutt, New Zealand

$$
\begin{array}{r}
\text { ₹: }+64-4-5704647 \\
\text { Fax: }+64-4-5704657
\end{array}
$$

Email: r.sparks@gns.cri.nz

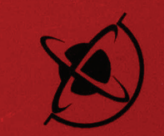

GEOLOGICAL \& NUCLEAR SCIENCES 